\title{
Extremal Dependence Modeling with Spatial and Survival Distributions
}

\author{
Diakarya Barro ${ }^{1}$ \\ ${ }^{1}$ Université Ouaga II BP: 417 Ouagadougou 12, Burkina Faso \\ Correspondence: Diakarya Barro, Université Ouaga II BP: 417 Ouagadougou 12, Burkina Faso. E-mail: dbarro2@ gmail.com
}

Received: November 11, 2016 Accepted: January 5, 2017 Online Published: January 23, 2017

doi:10.5539/jmr.v9n1p127～URL: http://dx.doi.org/10.5539/jmr.v9n1p127

\begin{abstract}
This paper investigates some properties of dependence of extreme values distributions both in survival and spatial context. Specifically, we prospose a spatial Extremal dependence coefficient for survival distributions. Madogram is characterized in bivariate case and multivariate survival function and the underlying hazard distributions are given in a risky context.
\end{abstract}

Keywords: survival distribution, extreme values distributions, variogram, spatial process, hazard function

2010 Math. Subject Classification: 62H20, 62H11, 60G15

\section{Introduction}

Extreme values (EV) analysis finds wide applications in many areas including climatology, environment sciences (Beirlant, J., et al., 2005), risk management (Balkema, G. \& Paul, E., 2007; Degen, M. \& Embrechts, P., 2008) and survival analysis (Hougaard, P., 2000). The distributions of this domain can be obtained as limiting distributions of properly normalized maxima of independent and identically distributed random variables. In particular if $Z=\left\{Z_{x} ; x \in \mathbb{R}^{2}\right\}$ is a max stable random field defined on a set $X=\left\{x_{1}, \ldots, x_{k}\right\}$, then the spatial EV analysis shows that $\mathrm{Z}$ results from observations of a stochasltic process such as

$$
Z(s)=\lim _{n \rightarrow \infty}\left\{\max _{1 \leq i \leq n}\left[\frac{x_{i}(s)-b_{n}(s)}{a_{n}(s)}\right]\right\} \text { with } s \in D
$$

provided the limit exists, where $\left\{a_{n}()>.0 ; n \geq 1\right\}$ and $\left\{b_{n}(),. ; n \geq 1\right\}$ are sequences of real constants, $s$ being a spatial location of a domain $D \subset \mathbb{R}^{d}$ and $Z(s)$, a random quantity (Padoan, S. A., et al., 2010).

Survival analysis is a subdomain of statistics which deals with failure or death time or natural catastroph. It is a important topic in many areas including biomedical, biostatistics, environment, etc (Padoan, S. A., et al., 2010; Resnick, S. I., 2008). One may distinguish three kind of models in survival analysis: the non parametric models, the semi-parametric models and the parametric ones.

Let $T=\left(T_{1}, \ldots, T_{n}\right)$ be a vector of lifetimes of $\mathrm{n}$ individuals in a given population with distribution $F_{T}$. If in particular T describes the life long time, the fraction of the population which will survive past a given vector of times $t=\left(t_{1}, \ldots, t_{n}\right)$ is provided by the survival distribution, conventionally denoted $\mathrm{S}_{T}$, such as

$$
S_{T}\left(t_{1}, \ldots, t_{n}\right)=\bar{F}_{T}\left(t_{1}, \ldots, t_{n}\right)=P\left(T_{1} \geq t_{1}, \ldots, T_{n} \geq t_{n}\right)
$$

The hazard function $h_{T}$ of $\mathrm{T}$ specifies the instantaneous rate of failure (risk or mortality rate) at a given date $t$ given that the individual survived up to time $t$. If the margins are absolutly continuous the cumulative density function (cdf) is also related to $\mathrm{S}_{T}$ such as

$$
h_{T}\left(t_{1}, \ldots, t_{n}\right)=\frac{f_{T}\left(t_{1}, \ldots, t_{n}\right)}{S_{T}\left(t_{1}, \ldots, t_{n}\right)} \text { and } f_{T}\left(t_{1}, \ldots, t_{n}\right)=(-1)^{n} \frac{\partial^{n} S\left(t_{1}, \ldots, t_{n}\right)}{\partial t_{1} \ldots \partial t_{n}} .
$$

Spatial analysis is a key component of statistic involving a collected from different locations. In particular, while studying in biostatistics, epidemiology, environment sciences, data have a common, that they are collected from different spatial locations and they are nether independent nor identically distributed. So, that in spatial framework, when survival times are spatially referenced, some of clusters of high or low times might be apparent on a visual inspection of the data. The question which naturally arises as to whether these observed spatial survival patterns can be explained by incorporating appropriate covariates into the model or whether, in order the unexplained spatial variation.

The main contribution of this paper is to investigate some asymptotic properties of multivariate dependence models both in survival and spatial context. Section 2 deals with spatial measures of extremal dependence. In particular the extremal 
dependance spatial coefficient is modeled and survival madogram is characterized in bivariate case. In Section 3, the survival and hazard distributions are given in a risky context.

\section{Survival Framework for Modeling Spatial Extremal Dependance}

\subsection{Spatial Extremal Dependence Coefficient}

In multivariate extreme values (EV) analysis, many related measures have been proposed for quantifying the magnitude of the extremal dependence when the random vector exhibits asymptotic dependence. In particular, in univariate EV study, even in spatial and survival framework, three types of distributions can summary the asymptotic behavior of conveniently normalized maximum of distributions (Beirlant, J., et al., 2005).

For a fixed $\mathrm{k}$ in $\mathbb{N}^{*}$ let $Y=\left(Y_{k, 1} ; \ldots ; Y_{k, s}\right)$ denote independent copies of stochastic process observed at given ereas s of a domain $\mathrm{S}$. Assume that the process $\{Y(s), s \in S\}$ is parametric max-stable distribution. Then the asymptotic distribution modeling the stochastic behavior is the same type like one of the three extremal spatial distributions

$$
F\left(y_{i}\left(s_{i}\right)\right)=\left\{\begin{array}{ll}
\exp \left\{-\exp \left(-y_{i}\left(s_{i}\right)\right)\right\} & =\Lambda\left(y_{i}\left(s_{i}\right)\right) ; y_{i}\left(s_{i}\right) \in \mathbb{R},(\text { Gumbel }) \\
\exp \left\{-\left(y_{i}\left(s_{i}\right)\right)^{-\theta}\right\} & =\Phi_{\theta}\left(y_{i}\left(s_{i}\right)\right) ; y_{i}\left(s_{i}\right)>0 ; \text { (Fréchet) } \\
\exp \left(-\left(-y_{i}\left(s_{i}\right)\right)^{\theta}\right) & =\Psi_{\theta}\left(y_{i}\left(s_{i}\right)\right) ; y_{i}\left(s_{i}\right) \leq 0, \text { (Weibull) }
\end{array} .\right.
$$

Let $T_{s}=\left(T_{1}(s), \ldots, T_{n}(s)\right)$ be a vector of lifetimes of $\mathrm{n}$ individuals in a given population observed at a given site $\mathrm{s}$ of spatial domain $S=\left\{\left(s_{1}, \ldots, s_{m}\right), s_{j} \in \mathbb{R}^{2}\right\}$. The process $T_{s}$ is the survival and stochastic random vector which with joint distribution $F_{s}=\left(F_{s, 1} ; \ldots ; F_{s, n}\right)$. Therefore, for all realization $\mathrm{y}$,

$$
F_{s}(y)=\left(F_{1, s}\left(y_{1}\right), \ldots, F_{n, s}\left(y_{n}\right)\right)=\left(F_{1}\left(y_{1}\left(s_{1}\right)\right), \ldots, F_{n}\left(y_{n}\left(s_{n}\right)\right)\right)=F(y(s)) .
$$

In all this study, our key assumption is that the process $T_{s}$ is continuous, stationary and is max-stable with generalized Fréchet margins. So, for a given site $s$ in $S_{\xi}$

$$
S_{\xi}=\left\{s_{i} \in S ; \sigma_{i}\left(s_{i}\right)+\xi_{i}\left(s_{i}\right)\left(y_{i}\left(s_{i}\right)-\mu_{i}\left(s_{i}\right)\right)>0\right\} \subset S .
$$

where $u_{+}=\max (u, 0)$ and $\left\{\mu_{\chi}(x) \in \mathbb{R}\right\},\left\{\sigma_{\chi}(x)>0\right\}$ and $\xi_{\chi}(x) \in \mathbb{R}$,

$$
F_{\theta}\left(y_{i}\left(s_{i}\right)\right)=\Phi_{\theta}\left(y_{i}\left(s_{i}\right)\right)=\exp \left\{-\left(\frac{y_{i}\left(s_{i}\right)-\mu_{i}\left(s_{i}\right)}{\sigma_{i}\left(s_{i}\right)}\right)_{+}^{-\theta}\right\} ; \theta>0
$$

Notice that such an assumption implies no loss of generality since even in survival and space-varying context, every onedimensional EV distribution can be obtained by a functional transformation of another. In particular, if for a given site $\mathrm{s}$,

$$
Y\left(x_{i}\right) \sim \Phi_{\theta}\left(y_{i}\left(x_{i}\right)\right) \Longrightarrow Z\left(x_{i}\right)=\mu_{x_{i}}+\frac{\sigma_{x_{i}}}{\xi_{x_{i}}}\left[Y\left(x_{i}\right)^{\xi_{x_{i}}}-1\right] .
$$

Among measures of extremal dependence there are the extremal coefficient (Hougaard, P., 2000) or the madogram and its nested model the link between two sets of $\mathbb{R}^{d}$ (Cooley, D., et al., 2006). Moreover and for simplicity reason let's denote like in (Barro, D., et al., 2016) that: $\tilde{F}_{i}^{s_{j}}\left(x_{i}\right)=F_{i}\left(x_{i}\left(s_{j}\right)\right)$ and $\tilde{x}_{i}^{s_{j}}=x_{i}\left(s_{j}\right)$. Under the restriction to the simplest case where $F_{i}\left(x_{i}\left(s_{j}\right)\right)=0$ if $i \neq j$, the following result allows us to provide a characterisation of the spatial extremal dependence (SED) in a survival field.

Theorem 1 Let $T_{s}$ be a vector of lifetimes of $\mathrm{n}$ individuals in a given population with distribution $\tilde{F}^{s}$ satisfying the key assumption.

i) The one-dimensional marginal law $\left\{\tilde{F}_{i}^{s_{j}} ; 1 \leq i \leq n\right\}$ of $\tilde{\mathrm{F}}^{s}$ is a max-stable process, that is there exists survival parametric normalizing sequences $\left\{\sigma_{i}\left(s_{i}\right)>0\right\}$ and $\left\{\mu_{i}\left(s_{i}\right) \in \mathbb{R}\right\}$ and $\left\{\xi_{i}\left(x_{t}\right) \in \mathbb{R}\right\}$ such that, for all $\mathrm{i}, 1 \leq i \leq n$

$$
\left[\tilde{F}_{i}^{s_{j}}\left(\sigma_{n}^{i}\left(\tilde{x}_{i}^{s_{j}}\right)+\mu_{n}^{i}\left(s_{i}\right)\right)\right]_{n \rightarrow+\infty}^{n} \begin{cases}{\left[1+\xi_{i}\left(s_{i}\right)\left(\frac{\tilde{x}_{i}^{s_{i}}-\mu_{i}\left(s_{i}\right)}{\sigma_{i}\left(s_{i}\right)}\right)\right]_{+}^{\frac{-1}{\xi_{i}\left(s_{i}\right)}}} & \text { if } \xi_{i}\left(s_{i}\right) \neq 0 \\ \exp \left\{-\left(\frac{\tilde{x}_{i}^{s_{i}}-\mu_{i}\left(s_{i}\right)}{\sigma_{i}\left(s_{i}\right)}\right)\right\} & \text { if } \xi_{i}\left(s_{i}\right)=0\end{cases}
$$

on $D_{\xi}\left(s_{i}\right)=\left\{s_{i} \in S, \tilde{x}_{i}^{s_{i}}-\mu_{i}\left(s_{i}\right)>0\right\}$ 
ii) There exists spatio-survival parametric measure of probability $P_{\xi(s)}$ defined on $\mathbb{R}^{2} \times\{s\}$ and a non-decreasing function $g_{s}$ defined on $D_{\xi}$ such that the survival and spatial extremal coefficent $\theta_{s}\left(h_{i j}\right)$ of the process is given by

$$
P_{s_{i j}, \xi_{s}}=g\left[s_{i}, \theta_{s}\left(h_{i j}\right)\right] ;
$$

where $h_{i j}=\left|s_{i}-s_{j}\right|$ is the separating distance between these sites $\mathrm{s}_{i}$ and $s_{j}$.

Proof. By assumption the distribution function $T_{s}$ of the process is max-stable. So, for all site $s$, there exist vectors of constants $\left\{\sigma_{n, s}>0\right\}$ and $\left\{\mu_{n, s} \in \mathbb{R}\right\}$ such that,

$$
\lim _{n \rightarrow+\infty} P\left(\bigcap_{i=1}^{n}\left\{\frac{M_{i}\left(\tilde{x}_{i}^{s_{i}}\right)-\mu_{i}\left(s_{i}\right)}{\sigma_{i}\left(s_{i}\right)} \leq y_{i}\left(s_{i}\right)\right\}\right)=G_{i}\left(y_{i}\left(\tilde{x}_{i}^{s_{i}}\right)\right)
$$

where $\mathrm{G}_{i}$ is an $\mathrm{EV}$ distribution and $M_{i}\left(x_{i}\right)$ is the spatial survival vector of the maximum

$$
M_{i}\left(x_{i}^{s_{j}}\right)=\left(\max _{1 \leq \mathrm{j} \leq \mathrm{m}}\left(x_{i}^{s_{j}}\right)\right)^{T} .
$$

But since $\mathrm{F}_{i}$ is the marginal distribution of $\mathrm{T}_{s}$ then, it lies on the max-domain of attraction of $\mathrm{G}_{i}$. Thus, for all site $s_{i}$, the relation (2.5) is equivalent to the generalized EV model, given by

$$
\lim _{n \rightarrow+\infty}\left[\tilde{F}_{i}^{s_{j}}\left(\sigma_{n}^{i}\left(\tilde{x}_{i}^{s_{j}}\right)+\mu_{n}^{i}\left(s_{i}\right)\right)\right]^{n}=\left\{\begin{array}{ll}
{\left[1+\xi_{i}\left(s_{i}\right)\left(\frac{\tilde{x}_{i}^{s_{i}}-\mu_{i}\left(s_{i}\right)}{\sigma_{i}\left(s_{i}\right)}\right)\right]_{+}^{\frac{-1}{\xi_{i}\left(s_{i}\right)}}} & \text { if } \xi_{i}\left(s_{i}\right) \neq 0 \\
\exp \left\{-\left(\frac{\tilde{x}_{i}^{s_{i}}-\mu_{i}\left(s_{i}\right)}{\sigma_{i}\left(s_{i}\right)}\right)\right\} & \text { if } \xi_{i}\left(s_{i}\right)=0
\end{array} .\right.
$$

where $\left\{\sigma_{i}\left(s_{i}\right)>0\right\}$ and $\left\{\mu_{i}\left(s_{i}\right) \in \mathbb{R}\right\}$ and $\left\{\xi_{i}\left(x_{t}\right) \in \mathbb{R}\right\}$ such that, for all i,1 $\leq i \leq n$ are respectively the spatio-survival parameters of location, scale and shape of the observation at the parametric site $\mathrm{s}_{i}$.

ii) In bivariate case and for all pair of sites $s_{i}$ and $s_{j}$ the extremal dependence parametric coefficient $\theta\left(s_{i}, s_{j}\right)=\theta_{i j}=\theta\left(h_{i j}\right)$ depends on the of separating distance $\mathrm{h}_{i j}$.

It follows that

$$
P\left[\tilde{F}^{s}\left(\tilde{x}_{i}^{s_{i}}\right) \leq y ; \tilde{F}^{s}\left(\tilde{x}_{j}^{s_{j}}\right) \leq y\right]=\exp \left[\frac{-\theta\left(h_{i j}\right)}{y}\right] .
$$

Moreover, using in the relation (2.6) the general form of a univariate EV model with normalizing coefficients $\sigma>0$, $\mu \in \mathbb{R}, \xi_{i}\left(s_{i}\right) \in \mathbb{R}$, it comes, in the particular bivariate context, that

$$
P\left(\tilde{F}^{s}\left(\tilde{x}_{i}^{s_{j}}\right) \leq y, \tilde{F}^{s}\left(\tilde{x}_{i}^{s_{j}}\right) \leq y\right)=\exp \left[-\theta\left(h_{i j}\right)\left(\left[1+\xi\left(\frac{\tilde{x}_{i}^{s_{i}}-\mu_{i}\left(s_{i}\right)}{\sigma_{i}\left(s_{i}\right)}\right)\right]_{+}^{\frac{1}{\xi_{i}\left(s_{i}\right)}}\right)\right] .
$$

Then, by introducing the concept of probability measure the relation (2.7) is equivalent to

$$
P\left(\tilde{F}^{s}\left(\tilde{x}_{i}^{s_{j}}\right) \leq y, \tilde{F}^{s}\left(\tilde{x}_{i}^{s_{j}}\right) \leq y\right)=\exp \left[-\theta\left(h_{i j}\right)\left(\left[1+\xi\left(\frac{\tilde{x}_{i}^{s_{i}}-\mu_{i}\left(s_{i}\right)}{\sigma_{i}\left(s_{i}\right)}\right)\right]_{+}^{\frac{1}{\xi_{i}\left(s_{i}\right)}}\right)\right]=P_{s_{i j}, \xi_{s}}
$$

and finally

Thus we obtain (2.4) as asserted

$$
P_{s_{i j}, \xi_{s}}=g\left[s_{i}, \theta_{s}\left(h_{i j}\right)\right]
$$

The following proposition provides a consequence of theorem 1

Corollary 2 Let $\left\{T^{s} ; s \in S\right\}$ a spatial process satisfying the key assumption. Then, for all site $s_{i} \in S$

i) the marginal survival parametric extremal density $f_{\xi_{i}}$ is given by

$$
f_{\xi_{i}}\left(t_{i}\left(\tilde{x}_{i}^{s_{j}}\right)\right)=\left\{\begin{array}{ll}
\frac{t_{i}\left(\tilde{x}_{i}^{s_{i}}\right)\left(1+\xi \log \left(t_{i}\left(\tilde{x}_{i}^{s_{i}}\right)\right)\right)^{1+\frac{1}{\xi_{i}\left(s_{i}\right)}}}{\exp \left[-\left(1+\xi \log \left(t_{i}\left(\tilde{x}_{i}^{s_{i}}\right)\right)\right)^{\frac{1}{\xi_{i}\left(s_{i}\right)}}\right]} & \text { if } \xi_{s_{j}} \neq 0 \\
\frac{1}{t_{i}^{2}\left(\tilde{x}_{i}^{s_{i}}\right)} \exp \left(\frac{-1}{t_{i}\left(\tilde{x}_{i}^{s_{i}}\right)}\right) & \text { if } \xi_{s_{j}}=0
\end{array} .\right.
$$


ii) the parametric hazard functions $h_{\xi}(t)$ are given

$$
h_{\xi_{i}}(t)=\left\{\begin{array}{ll}
\frac{\left\{\exp \left(\left(1+\xi \log \left(t_{i}\left(\tilde{x}_{i}^{s_{i}}\right)\right)\right)^{-\frac{1}{\xi_{i}\left(s_{i}\right)}}\right)-1\right\}}{t_{i}\left(\tilde{x}_{i}^{s_{i}}\right)\left(1+\xi \log \left(t_{i}\left(\tilde{x}_{i}^{s_{i}}\right)\right)\right)^{\frac{1}{\xi_{i}\left(s_{i}\right)}+1}} & \text { if } \xi_{s_{j}} \neq 0 \\
\frac{1}{t_{i}\left(x_{i}\left(s_{i}\right)\right)^{2}\left(\exp \left(\frac{1}{t_{i}\left(\tilde{x}_{i}^{s_{i}}\right)}\right)-1\right)} & \text { if } \xi_{s_{j}}=0
\end{array} .\right.
$$

Proof. In such a case, the parametric survival function

$$
S_{\xi}\left(t_{1}, \ldots, t_{n}\right)=P_{\xi}\left(T_{1} \geq t_{1}, \ldots, T_{n} \geq t_{n}\right)
$$

is given marginally by

$$
S_{\xi}\left(t_{i}\right)=\left\{\begin{array}{ll}
1-\exp \left[-\left(1+\xi \log t_{i}\right)^{-\frac{1}{\xi_{i}\left(s_{i}\right)}}\right] & \text { if } \xi_{i}\left(s_{i}\right) \neq 0 \\
1-\exp \left(-\frac{1}{t_{i}}\right) & \text { if } \xi_{i}\left(s_{i}\right)=0
\end{array} .\right.
$$

Hence, the hazard function $h_{\xi}(t)=\frac{f_{\xi}(t)}{S_{t}(t)}$ is given by

$$
h_{\xi}(t)= \begin{cases}\frac{1}{t(1+\xi \log t)^{\frac{1}{\xi}+1}\left\{\exp \left((1+\xi \log t)^{-\frac{1}{\xi}}\right)-1\right\}} & \text { if } \xi_{i}\left(s_{i}\right) \neq 0 \\ \frac{1}{t^{2}\left(\exp \left(\frac{1}{t}\right)-1\right)} & \text { if } \xi_{i}\left(s_{i}\right)=0\end{cases}
$$

\subsection{Distortional Function of Spatial Extremal Model}

The following result characterizes a multivariate survival distribution via a spatial and distortional measure of dependence.

Proposition 3 Let $\left\{T^{s} ; s \in S\right\}$ a spatial process satisfying the key assumption. Then there exists a spatial conditional dependence measure such as $D_{s}$ defined on the spatial unit simplex, for all $\tilde{x}^{s}=\left(\tilde{x}_{1}^{s_{1}}, \ldots, \tilde{x}_{n}^{s_{n}}\right) \in \overline{\mathbb{R}}^{n}$;

$$
\widetilde{S}_{n}^{s}=\left\{\left(t_{1}(s) \ldots, t_{n}(s)\right) \in[0,1]^{n} ; \sum_{i=1}^{n} t_{i}(s)=1\right\}
$$

such that,

$$
\tilde{F}^{s}\left(\tilde{x}^{s}\right)=1-\exp \left\{\sum_{i=1}^{n} \frac{t_{i}\left(\tilde{x}_{i}^{s_{i}}\right)}{\left(1+\xi \log \left(t_{i}\left(\tilde{x}_{i}^{s_{i}}\right)\right)\right)^{1+\frac{1}{\xi}}} D_{s}\left(\frac{\tilde{x}_{i}^{s_{i}}}{\sum_{i=1}^{n} \tilde{x}_{i}^{s_{i}}}, \ldots, \frac{\tilde{x}_{m-1}^{s_{m-1}}}{\sum_{i=1}^{n} \tilde{x}_{i}^{s_{i}}}\right)\right\} .
$$

Proof. The EV analysis results from asymptotic normalized vector of maxima of a random vector which converges to a non degenerated multivariate EV model G. One of extremal study approach is the Peacks-over threshold (POT). Then the vector of exceedances of the same sample have a generalized Pareto model $\mathrm{H}$.

Particularly if the extremal function $\tilde{F}^{s}$ underlying the survival process $T_{S}$. It follows that its spatio-survival associated POT model $\tilde{\mathrm{H}}^{s}$ satisfies, for all $\tilde{x}^{s}=\left(\tilde{x}_{1}^{s_{1}}, \ldots, \tilde{x}_{n}^{s_{n}}\right) \in \overline{\mathbb{R}}^{n}$, the relationship by

$$
H\left(x^{s}\right)=1+\left(\sum_{i=1}^{n} \tilde{x}_{i}^{s_{i}}\right) \tilde{A}_{s}\left(\frac{\tilde{x}_{1}^{s 1}}{\sum_{i=1}^{n} \tilde{x}_{i}^{s_{i}}}, \ldots, \frac{\tilde{x}_{m-1}^{s_{m-1}}}{\sum_{i=1}^{n} \tilde{x}_{i}^{s_{i}}}\right)=1+\log F\left(\tilde{x}^{s}\right) ;
$$

where $\tilde{A}_{F}$ is a spatio-survival dependence function of Pickands associated to $\tilde{F}$.

Furthermore, for a given $1<N<n$ let consider the N-partition of the spatial domain $S$ proposed in [9]

$$
S=\left\{\left(s_{1}, \ldots, s_{m}\right), s_{j} \in \mathbb{R}^{2}\right\}=S_{N} \cup S_{\bar{N}} .
$$

Then, it follows that the corresponding distorsional probability $\tilde{\delta}_{s}$ is such that;

$$
\tilde{\delta}_{s}(x)=\tilde{\delta}\left(\tilde{x}_{1}^{s 1}, . . \tilde{x}_{n}^{s_{i}}\right)=1-\frac{P\left(T_{j} \leq \tilde{x}_{j}^{s_{j}} ; N \leq j \leq n\right)}{P\left(T_{i} \leq x_{i} ; 1 \leq \mathrm{i} \leq \mathrm{N}-1\right)} .
$$


Moreover let $\tilde{F}_{N}^{s}$ and $\tilde{F}_{\bar{N}}^{s}$ be the corresponding partitional distributions functions. So, it comes that

$$
\tilde{\delta}\left(\tilde{x}_{1}^{s 1}, \ldots \tilde{x}_{n}^{s_{i}}\right)=1-\frac{\tilde{F}_{\bar{N}}^{s}\left(\tilde{x}_{N}^{s_{N}} ; \ldots ; \tilde{x}_{n}^{s_{i}}\right)}{\tilde{F}_{N}^{s}\left(\tilde{x}_{1}^{s 1} ; \ldots ; \tilde{x}_{N-1}^{s_{i}}\right)} .
$$

Furthermore, from results due to Dossou et al. (Dossou,-G. S., 2009) both the partitional distributions functions $\tilde{F}_{N}^{s}$ and $\tilde{F}_{\bar{N}}^{s}$ lie also in the max-domain of attraction of two multivariate EV distribtutions. And by noting $\tilde{A}_{N}$ and $\tilde{A}_{\bar{N}}$ the Pickands dependence functions of $\mathrm{t} \tilde{F}_{N}^{s}$ and $\tilde{F}_{\bar{N}}^{s}$ respectively, it come that, even in a spatio-survival context

$$
\begin{aligned}
& \tilde{\delta}\left(\tilde{x}_{1}^{s 1}, \ldots, \tilde{x}_{n}^{s_{i}}\right)=\exp \left\{-\left(\sum_{i=1}^{n} \tilde{x}_{i}^{s_{i}}\right) \tilde{A}_{s}\left(\frac{\tilde{x}_{1}^{s 1}}{\sum_{i=1}^{n} \tilde{x}_{i}^{x_{i}}}, \ldots, \frac{\tilde{x}_{m-1}^{s_{m-1}}}{\sum_{i=1}^{n} \tilde{x}_{i}^{s_{i}}}\right)+\right. \\
& \left.\left(\sum_{i=1}^{n} \tilde{x}_{i}^{s_{i}}\right) \tilde{A}_{s}\left(\frac{\tilde{x}_{1}^{s 1}}{\sum_{i=1}^{n} \tilde{x}_{i}^{s_{i}}}, \ldots, \frac{\tilde{x}_{m-1}^{s_{m-1}}}{\sum_{i=1}^{n} \tilde{x}_{i}^{s_{i}}}\right)\right\}
\end{aligned}
$$

Which can be written equivalently,

$$
\tilde{\delta}\left(\tilde{x}_{1}^{s 1}, \ldots \tilde{x}_{n}^{s_{i}}\right)=\exp \left\{-\left(\sum_{i=1}^{n} \tilde{x}_{i}^{s_{i}}\right) \tilde{D}_{s}\left(\frac{\tilde{x}_{1}^{s 1}}{\sum_{i=1}^{n} \tilde{x}_{i}^{s_{i}}}, \ldots, \frac{\tilde{x}_{m-1}^{s_{m-1}}}{\sum_{i=1}^{n} \tilde{x}_{i}^{s_{i}}}\right)\right\}
$$

where $\tilde{D}_{s}$ being a distortional spatial and survival dependence function

$$
D\left(t\left(\tilde{x}^{s}\right)\right)=A\left(t_{1}\left(\tilde{x}_{1}^{s_{1}}\right), \ldots, t_{m-1}\left(\tilde{x}_{m-1}^{s_{m-1}}\right)\right)+\left(1-t_{1}\left(\tilde{x}_{1}^{s_{1}}\right)\right) A_{\bar{N}_{1}}\left(\frac{t_{2}\left(\tilde{x}_{2}^{s_{1}}\right)}{1-t_{2}\left(\tilde{x}_{2}^{s_{1}}\right)}, \ldots, \frac{t_{m-1}}{1-t_{2}\left(\tilde{x}_{2}^{s}\right)}\right)
$$

Particularly in bivariate case it is easy to show that $D\left(t\left(\tilde{x}^{s}\right)\right)$ is defined from $\mathbb{R}^{+}$to $\left[\frac{-1}{2}, 1\right]$ by

$$
D\left(t\left(\tilde{x}^{s}\right)\right)=A\left(\frac{1}{\left.1-t\left(\tilde{x}^{s}\right)\right)}\right)-\frac{t}{1+t\left(\tilde{x}^{s}\right)} \text {. }
$$

Specially, for the logistic model:

$$
F_{\theta}^{\check{s}}\left(\tilde{x}_{1}^{s_{i}}, \tilde{x}_{2}^{s_{i}}\right)=\exp \left\{-\left(\left(\tilde{x}_{1}^{s_{i}}\right)^{\theta}+\left(\tilde{x}_{1}^{s_{i}}, \tilde{x}_{2}^{s_{i}}\right)^{\theta}\right)^{\frac{1}{\theta}}\right\}
$$

the spatial conditional measure is

$$
D_{\theta, t}^{\check{s}}(x)=\frac{x_{t}}{1+x_{t}}\left[\left(1+x_{t}^{-\theta}\right)^{\frac{1}{\theta}}-1\right]
$$

which is given graphically has follows

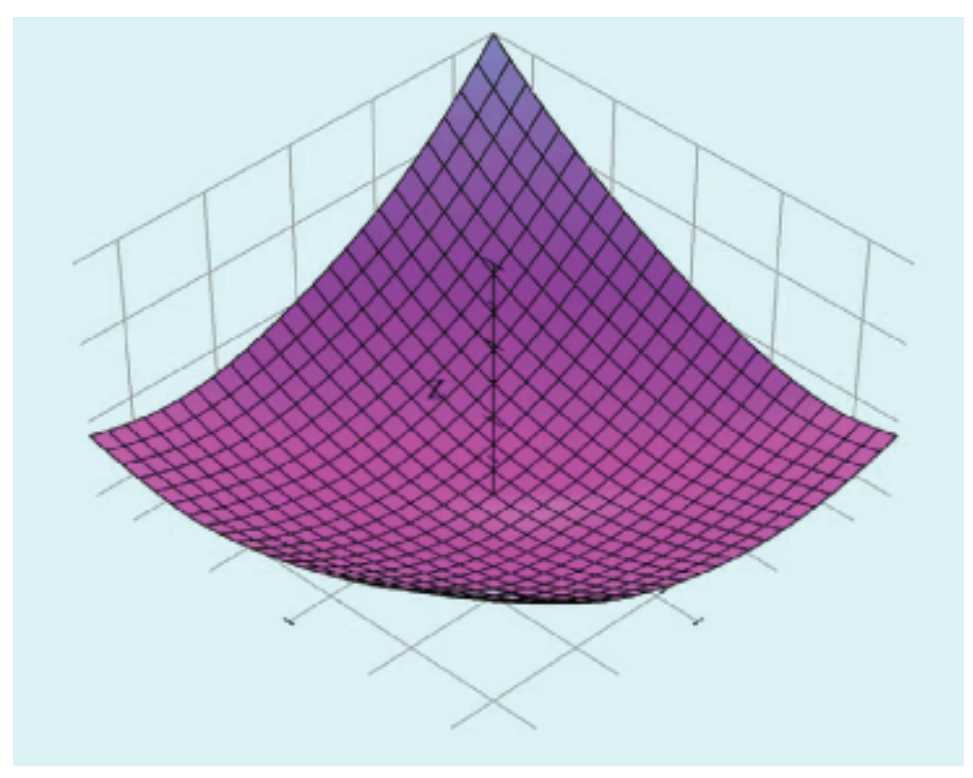

Figure 1. Bivariate logistic model for $\theta_{1}=\theta_{2}=2$ 


\subsection{A New Charaterization of Survival Madogram}

Madogram is a measure of the full pairwise extremal dependence function to evaluate dependence among extreme regional observation. Some extensions of this tool have been proposed. Specifially while modeling spatial extreme variablity of an isotropic and max-stable field, Cooley (Cooley, D., et al., 2006) proposed the F-madogram $\gamma_{F}(h)$ which transforms the process via its marginal F. The following result provides a parametrization of characterizing of the madogram.

Proposition 4 Let $\left\{T^{s} ; s \in S\right\}$ a spatial process satisfying the key assumption with distribution $\tilde{F}^{s}$. Then, the survival $\lambda$-madogram associated to the bivariate margins of $\mathrm{F}$ is given by the ratio

$$
\gamma_{\lambda}(h)=\frac{P\left(D_{h}, \lambda, s_{i}\right)}{Q\left(D_{h}(\lambda, 1-\lambda)+\lambda\right)} ;
$$

where $\mathrm{P}$ and $\mathrm{Q}$ are convenient polynoms and $D_{h}$ being a distortional spatial dependence measure.

Proof. In the previous proposition, the bivariate case implies that, particularly in bivariate case it is easy to shows that $D\left(t\left(\tilde{x}^{s}\right)\right)$ is defined from $\mathbb{R}^{+}$to $\left[\frac{-1}{2}, 1\right]$ by

$$
D\left(t\left(\tilde{x}^{s}\right)\right)=A\left(\frac{1}{\left.1-t\left(\tilde{x}^{s}\right)\right)}\right)-\frac{t}{1+t\left(\tilde{x}^{s}\right)} .
$$

Furthermore, in condional study, Proposition 6 of the paper (Barro, D. et al., 2012) provides that, under additional contraints, the $\lambda$-madogram can be expressed as

$$
\gamma_{\lambda}(h)=\frac{1}{D_{h}(\lambda, 1-\lambda)+\lambda}-c(\lambda) \text { with } c(\lambda)=\frac{2 \lambda(1-\lambda)+1}{2(\lambda+1)(2-\lambda)}
$$

where $D_{h}$ is a conditional spatial measure convex defined on the unit simplex of $\mathbb{R}^{2}$.

In our context by replacing $D\left(t\left(\tilde{x}^{s}\right)\right)$ by $D\left(t\left(\tilde{x}^{s}\right)\right)$ it follows easily that

$$
\gamma_{\lambda_{s}}(h)=\frac{-[2 \lambda(1-\lambda 1)+1]\left(D_{h}(\lambda, 1-\lambda)+\lambda\right) 2(\lambda+1)(2-\lambda)}{\left(D_{h}(\lambda, 1-\lambda)+\lambda\right)[2(\lambda+1)(2-\lambda)]}=\frac{P\left(D_{h}, \lambda, s_{i}\right)}{Q\left(D_{h}(\lambda, 1-\lambda)+\lambda\right)}
$$

where $D_{h}$ is a conditional spatial measure convex defined on the unit simplex of $\mathbb{R} 2$



Figure 2. Bivariate distortional $\lambda$ - madogram 


\section{Survival and Hazard Distributions in a Risky Context}

In epidemiological studies, the intensity of contamination must change over time. For example, at begining of the epidemy, the intensity is high but it decreases when the sanitaries autorities take some dispositions epidemy.

Let $T_{s}=\left(T_{1}(s), \ldots, T_{n}(s)\right)$ be the survival, continuous and stochastic random vector $\mathrm{T}$ satisfying the key assumption. Consider $H$ as a closed half space in $\mathbb{R}^{n}$ where $P\left(T_{s} \in H\right)>0$. As in the high scenarios defined by Degen (see [1], [5] and [6]), the following results characterize the spatial and survival risk and some derivative properties.

Definition The spatial and survival high risk scenario $T_{s}^{H}$ associated to the process $\mathrm{T}_{s}$ is defined as the vector $\mathrm{T}(s)$ conditioned to lie in the half space $H$. The spatial probability distribution function $(p d f) F_{T_{s}}^{H}$ of $T_{s}^{H}$ on $\mathbb{R}^{2}$ is such that $F_{T_{s}}^{H}=P \circ T_{s}^{-1}$., then $Z_{s}^{H}$ has the high risk distribution $\pi^{H}$ given by

$$
d F_{T_{s}}^{H}(z(s))=\delta_{H}(z(s)) d \pi(z(s)) / \pi(H) .
$$

One obtains the following relation between $\mathrm{T}$ and its canical parametrisation in a risky context.

Proposition If $\mathrm{T}_{s}$ has the distribution $\tilde{F}_{s}$, then $\mathrm{T}_{s}^{H}$ has the high risk distribution $\tilde{F}_{s}^{H}$ given by, for all $i=1, \ldots, m$.

$$
\tilde{F}_{s}^{H}\left(t_{1}, \ldots, t_{d}\right)=\tilde{f}_{s}^{H}\left(\tilde{t}_{s, 1}, \ldots, \tilde{t}_{s, d}\right) \prod_{i=1}^{d} \delta_{H_{i}}\left(t_{i}\right) \tilde{F}_{1, s}^{H}\left(t_{1}\right) / \pi_{i}\left(F_{i}\right)
$$

where, $\tilde{t}_{s, i}=\tilde{F}_{i, s}^{H}\left(t_{i}\right)$ are the marginals distributions functions of the distribution $\mathrm{T}^{H}$ and given

$$
\tilde{f}_{s}^{H}\left(u_{1}, \ldots, u_{m}\right)=\frac{f\left(F_{1}^{-1}\left(u_{1}\right), \ldots, F_{m}^{-1}\left(u_{m}\right)\right)}{f_{1}\left(F_{1}^{-1}\left(u_{1}\right), \ldots, f_{m}\left(F_{m}^{-1}\left(u_{m}\right)\right)\right.}
$$

for all $\left(u_{1}, \ldots, u_{m}\right) \in[0,1]^{m}$.

Proof. Let $\left(X_{1}, \ldots, X_{n}\right), n \in \mathbb{N}$, be a vector of random i.i.d variables with a joint distribution $\mathrm{F}$ with continuous margins $\mathrm{F}_{i}$. According to Sklar's theorem (see [15]), there exists a unique copula, $\mathrm{C}_{T}$ providing a canonical parameterisation of $\mathrm{F}$ via its univariate marginal quantile functions $F_{i}^{-1}$ such that,

$$
F_{i}^{-1}(u)=\inf \left\{x_{i} \in \mathbb{R}, F_{i}\left(x_{i}\right) \geq u\right\} ;
$$

for all $x=\left(x_{1}, \ldots, x_{m}\right) \in(\mathbb{R} \cup\{ \pm \infty\})^{m}$.

$$
F\left(x_{1}, \ldots, x_{m}\right)=C_{T}\left[F_{1}\left(x_{1}\right), \ldots, F_{m}\left(x_{m}\right)\right] .
$$

Or conversively, for $\mathrm{C}_{F}$ providing a canonical parameterisation of $\mathrm{F}$ via its univariate marginal quantile functions $F_{i}^{-1}$ such that:

$$
C_{F}\left(u_{1}, \ldots, u_{n}\right)=F\left(F_{1}^{-1}\left(u_{1}\right), \ldots, F_{n}^{-1}\left(u_{n}\right)\right) .
$$

Differentiating the formula (3.4) shows that the density function of the copula is equal to the ratio of the joint density $\mathrm{h}$ of $\mathrm{H}$ to the product of marginal densities $h_{i}$ such as, for all $\left(u_{1}, \ldots, u_{n}\right) \in[0,1]^{n}$,

$$
c\left(u_{1}, \ldots, u_{n}\right)=\frac{\partial^{n} C\left(u_{1}, \ldots, u_{n}\right)}{\partial u_{1} \ldots \partial u_{m}}=\frac{f\left[F_{1}^{-1}\left(u_{1}\right), \ldots, F_{n}^{-1}\left(u_{n}\right)\right]}{f_{1}\left[F_{1}^{-1}\left(u_{1}\right)\right] \times \ldots \times h_{n}\left[F_{n}^{-1}\left(u_{n}\right)\right]} .
$$

Let $Z$ be a random vector in $\mathbb{R}^{m}$ and $H=H_{1} \times \ldots \times H_{m} \subset \mathbb{R}^{m}$ a closed half space with $P(Z \in H)>0$. Let's suppose that $z_{i} \in \mathbf{N}=H_{1} \cap \ldots \cap H_{d}$, for all $i=1, \ldots, m$. If the marginals distributions of the high risk scenarios distribution $\pi^{H}$ are all continuous, then the density of the $\tilde{\mathrm{F}}_{s}$ is given by

$$
c\left(u_{1}, \ldots, u_{n}\right)=\frac{\left.\delta_{H} d \pi\left(\tilde{u}_{1}^{H_{1}}, \ldots, \tilde{u}_{m}^{H_{m}}\right)\right) / \pi(H)}{d \pi_{1}\left(\tilde{u}_{1}^{H_{1}}\right) \times \ldots \times d \pi_{m}\left(\tilde{u}_{m}^{H_{m}}\right) /\left(\pi_{1}\left(H_{1}\right) \times \ldots \times \pi_{m}\left(H_{m}\right)\right)}
$$


where $\tilde{u}_{i}^{H_{i}}=\left(\pi_{i}^{H_{i}}\right)^{(-1)}\left(u_{i}\right)$, the inverses of the marginals distributions $\left(\pi_{i}^{H_{i}}\right)$. Suppose that the high risk scenarios margins $\pi_{1}^{H_{1}}, \ldots, \pi_{d}^{H_{d}}$ are continuous. Using the relation, it comes that

$$
c\left(\pi_{1}^{H_{1}}\left(z_{1}\right), \ldots, \pi_{d}^{H_{d}}\left(z_{d}\right)\right)=\frac{d \pi^{H}\left(z_{1}, \ldots, z_{d}\right)}{\prod_{i=1}^{d} \delta_{H_{i}}\left(z_{i}\right) d \pi_{i}\left(z_{i}\right) / \pi_{i}\left(H_{i}\right)}
$$

If $z_{i} \in \boldsymbol{\aleph}, \forall i=1, \ldots, d$ then we get this relation :

$$
c\left(u_{1}, \ldots, u_{d}\right)=\frac{\left.d \pi^{H}\left(\tilde{u}_{i}^{H_{i}}, \ldots, \tilde{u}_{d}^{H_{d}}\right)\right)}{\prod_{i=1}^{d} \delta_{H_{i}}\left(z_{i}\right) d \pi_{i}\left(z_{i}\right) / \pi_{i}\left(H_{i}\right)}
$$

where $\pi_{i}^{H_{i}}\left(z_{i}\right)=u_{i}$ or $z_{i}=\left(\pi_{i}^{H_{i}}\right)^{(-1)}\left(u_{i}\right)$.

This gives

$$
c\left(u_{1}, \ldots, u_{d}\right)=\frac{\delta_{H} d \pi\left(\tilde{u}_{1}^{H_{1}}, \ldots, \tilde{u}_{m}^{H_{m}}\right) / \pi(H)}{\delta_{\aleph} d \pi_{1}\left(\tilde{u}_{1}^{H_{1}}\right) \times \ldots \times d \pi_{d}\left(\tilde{u}_{m}^{H_{m}}\right) /\left(\pi_{1}\left(H_{1}\right) \times \ldots \times \pi_{m}\left(H_{m}\right)\right)}
$$

Since $\delta_{\mathfrak{\aleph}}=1$, then it follows that $z_{i} \in \mathbf{\aleph}=\bigcap_{i=1}^{d} H_{i}$.

Finally we obtain

$$
\tilde{f}_{s}^{H}\left(u_{1}, \ldots, u_{m}\right)=\frac{f\left(F_{1}^{-1}\left(u_{1}\right), \ldots, F_{m}^{-1}\left(u_{m}\right)\right)}{f_{1}\left(F_{1}^{-1}\left(u_{1}\right), \ldots, f_{m}\left(F_{m}^{-1}\left(u_{m}\right)\right)\right.}
$$

Thus, we obtain the relation (3.3) as asserted

\section{Conclusion}

The results of this study show that the survial and spatial framework are also convenient to model extremal dependence. Tools of dependence such as the extremal dependance coefficient, the multivariate dependence function, the madogram have been modeled both in spatial and survival context. The survival and hazard distributions are given in a risky context.

\section{References}

Balkema, G., \& Paul, E. (2007). High risk scenarios and extreme: a geometric approach.

Barro, D. (2009). Conditional Dependence of Trivariate Generalized Pareto Distributions, Asian Journal of Mathematics and Statistics, 2(3), 41-54. http://dx.doi.org/10.3923/ajms.2009.41.54

Barro, D., et al. (2012). Spatial Stochastic Framework For Sampling Time Parametric Max-stable Processes. Inter.JSP, 1(2), 203-210. http://dx.doi.org/10.5539/ijsp.v1n2p203

Barro, D., et al. (2016). Spatial Tail Dependence and Survival Stability in a Class of Archimedean Copulas.International Journal of Mathematics and Mathematical Sciences, (8). http://dx.doi.org/10.1155/2016/8927248

Benjamim, M. T. (2015). Auxiliary Varables Markov Chain monte Carlo for spatial survival geostatistical Model,

Beirlant, J., Goegebeur, Y., Segers, J., \& Teugels, J. (2005). Statistics of Extremes : Theory and Applications. John Wiley and Sons, Chichester.

Cooley, D., Naveau, P., Jomelli, V., Rabatel, A., \& Grancher, D., (2006). A Bayesian hierarchical extreme value model for lichenometry. Environmetrics, 17, 555-574. http://dx.doi.org/10.1002/env.764

Degen, M., E. (2008). EVT-based estimation of risk capital and convergence of high quantiles. Advances in Applied Probability, 40(3), 696-715.

Degen, M. (2006). On Multivariate Generalised Pareto Distributions and High Risk Scenarios. Department of Mathematics, ETH Zü rich, Diploma thesis. http://www.math.ethz.ch/ degen/thesis.pdf

Dossou, -G., S., (2009). Modelling the Dependence of Parametric Bivariate Extreme Value Copulas. Asian Journal of Mathematics and Statistics, 2(3), 41-54. http://dx.doi.org/10.3923/ajms.2009.41.54

Fonseca, et al., (2012). Generalized madogram and pairwise dependence of maxima over two regions of a random field.

Hougaard, P. (2000). Analysis for Multivariate Survival Data, Statistics for Biology and Health. Springer-Verlag, New York, Hall, London. 
Nelsen, R. B. (1999). An Introduction to copulas. Lectures notes in Statistics 139, Springer-Verlag.

Padoan, S. A., Ribatet, M., \& Sisson, S. A. (2010). Likelihood-Based Inference for Max-Stable Processes, Journal of the American Statistical Association, 105(489), Theory and Methods. http://dx.doi.org/10.1198/jasa.2009.tm08577

Resnick, S. I. (2008). Extreme Values, Regular Variation, and Point Processes (reprint). Springer, NY.

Tajvidi, N. (1996). Characterisation and Some Statistical Aspects of Univariate and Multivariate Generalised Pareto Distributions. Dissertation, Department of Mathematics, Chalmers.

\section{Copyrights}

Copyright for this article is retained by the author(s), with first publication rights granted to the journal.

This is an open-access article distributed under the terms and conditions of the Creative Commons Attribution license (http://creativecommons.org/licenses/by/4.0/). 